\title{
Detection of type, duration, and intensity of physical activity using an accelerometer
}

Citation for published version (APA):

Bonomi, A., Goris, A. H., Yin, B., \& Westerterp, K. R. (2009). Detection of type, duration, and intensity of physical activity using an accelerometer. Medicine and Science in Sports and Exercise, 41(9), 1770-7. https://doi.org/10.1249/MSS.0b013e3181a24536

Document status and date:

Published: 01/01/2009

DOI:

10.1249/MSS.0b013e3181a24536

Document Version:

Publisher's PDF, also known as Version of record

Document license:

Taverne

Please check the document version of this publication:

- A submitted manuscript is the version of the article upon submission and before peer-review. There can be important differences between the submitted version and the official published version of record.

People interested in the research are advised to contact the author for the final version of the publication, or visit the DOI to the publisher's website.

- The final author version and the galley proof are versions of the publication after peer review.

- The final published version features the final layout of the paper including the volume, issue and page numbers.

Link to publication

\footnotetext{
General rights rights.

- You may freely distribute the URL identifying the publication in the public portal. please follow below link for the End User Agreement:

www.umlib.nl/taverne-license

Take down policy

If you believe that this document breaches copyright please contact us at:

repository@maastrichtuniversity.nl

providing details and we will investigate your claim.
}

Copyright and moral rights for the publications made accessible in the public portal are retained by the authors and/or other copyright owners and it is a condition of accessing publications that users recognise and abide by the legal requirements associated with these

- Users may download and print one copy of any publication from the public portal for the purpose of private study or research.

- You may not further distribute the material or use it for any profit-making activity or commercial gain

If the publication is distributed under the terms of Article $25 \mathrm{fa}$ of the Dutch Copyright Act, indicated by the "Taverne" license above, 


\title{
Detection of Type, Duration, and Intensity of Physical Activity Using an Accelerometer
}

\author{
ALBERTO G. BONOMI ${ }^{1,2}$, ANNELIES H.C. GORIS ${ }^{3}$, BIN YIN $^{4}$, and KLAAS R. WESTERTERP ${ }^{1}$ \\ ${ }^{I}$ Department of Human Biology, Maastricht University, Maastricht, THE NETHERLANDS; ${ }^{2}$ Care and Health Applications, \\ Philips Research Laboratories, Eindhoven, THE NETHERLANDS; ${ }^{3}$ DirectLife, Philips New Wellness Solutions, \\ THE NETHERLANDS; and ${ }^{4}$ Biomedical Sensor Systems, Philips Research Laboratories, Eindhoven, THE NETHERLANDS
}

\begin{abstract}
BONOMI, A. G., A. H. GORIS, B. YIN, and K. R. WESTERTERP. Detection of Type, Duration, and Intensity of Physical Activity Using an Accelerometer. Med. Sci. Sports Exerc., Vol. 41, No. 9, pp. 1770-1777, 2009. Objective: The aim of this study was to develop models for the detection of type, duration, and intensity of human physical activity using one triaxial accelerometer. Methods: Twenty subjects ( $\mathrm{age}=29 \pm 6 \mathrm{yr}, \mathrm{BMI}=23.6 \pm 3.2 \mathrm{~kg} \cdot \mathrm{m}^{-2}$ ) performed 20 selected activities, including walking, running, and cycling, wearing one triaxial accelerometer mounted on the lower back. Identification of activity type was based on a decision tree. The decision tree evaluated attributes (features) of the acceleration signal. The features were measured in intervals of defined duration (segments). Segment size determined the time resolution of the decision tree to assess activity duration. Decision trees with a time resolution of 0.4, $0.8,1.6,3.2,6.4$, and $12.8 \mathrm{~s}$ were developed, and the respective classification performances were evaluated. Multiple linear regression was used to estimate speed of walking, running, and cycling based on acceleration features. Results: Maximal accuracy for the classification of activity type (93\%) was reached when the segment size of analysis was 6.4 or $12.8 \mathrm{~s}$. The smaller the segment size, the lower the classification accuracy achieved. Segments of $6.4 \mathrm{~s}$ gave the highest time resolution for measuring activity duration without decreasing the classification accuracy. The developed models estimated walking, running, and cycling speeds with a standard error of $0.20,1.26$, and $1.36 \mathrm{~km} \cdot \mathrm{h}^{-1}$, respectively. Conclusions: This study demonstrated the ability of a triaxial accelerometer in detecting type, duration, and intensity of physical activity using models based on acceleration features. Future studies are needed to validate the presented models in free-living conditions. Key Words: TRACMOR, DECISION TREE, CLASSIFICATION, AMBULATORY MONITORING
\end{abstract}

$\mathrm{P}$ hysical activity (PA) is recommended to improve health and to reduce risk for several diseases, such as cardiovascular diseases, diabetes mellitus type II, osteoporosis, obesity, and certain types of cancer $(3,11$, 14,17). For this reason, the objective assessment of PA in free-living conditions is an important component of many scientific investigations aimed at defining the effectiveness of intervention strategies to increase PA. Furthermore, measuring accurately pattern, duration, and intensity of PA is required to improve the understanding of individuals' behavior and to quantify the relation between PA and disease outcomes.

A variety of methods exist to objectively measure PA in daily life (20). Ideally, PA should be measured for a period

\footnotetext{
Address for correspondence: Alberto G. Bonomi, M.D., Department of Human Biology, Maastricht University, P.O. Box 616, 6200 MD Maastricht, The Netherlands; E-mail: a.bonomi@HB.unimaas.nl. Submitted for publication November 2008. Accepted for publication February 2009.

0195-9131/09/4109-1770/0

MEDICINE \& SCIENCE IN SPORTS \& EXERCISE ${ }_{\circledast}$ Copyright $(\subset 2009$ by the American College of Sports Medicine

DOI: 10.1249/MSS.0b013e3181a24536
}

representative of the habitual activity level, with minimum discomfort for the subject and using low-cost systems for implementation in large-scale studies. Activity monitors based on an accelerometer sensor reasonably satisfy these requirements, and therefore they have been widely used to monitor PA. Accelerometer output, as defined by arbitrary acceleration units (AAU) per minute, reflects pattern, duration, and intensity of PA, and it is used to estimate activity-related energy expenditure $(5,23)$. However, the description of PA by measuring AAU has limitations. AAU has limited ability to identify types of PA performed. This information is important because it may improve assessment of activity pattern and intensity. Furthermore, measuring PA in epochs of 1 min limits the time resolution for determining activity duration. Thus, improvements in the assessment of types, duration, and intensity of PA are necessary to correctly evaluate habitual PA.

In recent decades, accelerometer sensors have been adopted to identify human movements by using classification algorithms. Zhang et al. (29) proposed a method based on several accelerometers positioned in different body parts (chest, legs, and feet) to identify up to 32 movements and postures achieving a classification accuracy of $95 \%$. In other studies, multiple sensors have been used to identify several types of PA achieving a classification accuracy from 
$85 \%$ to $95 \%(2,10)$. More recently, identification of PA has been investigated using a single acceleration sensor, placed around the waist $(15,21)$ or on the chest $(8)$. Then, the overall classification accuracy was between $71 \%$ and $91 \%$ to identify postures, walking, and various sport activities such as rowing, running, and cycling. The most common classification algorithms are decision trees $(2,8)$, neural networks (29), Bayesian classifiers (16), and hidden Markov models (24). These algorithms identify activity types by evaluating attributes of the acceleration signal measured in portions of defined length (segments). A segment of the acceleration includes a certain number of data points determined by the sampling frequency of the signal and by the time length of the segment. Given a certain sampling frequency, the longer the segment size, the more samples are considered to calculate attributes (features) of the acceleration. Acceleration features are used to classify the type of activity performed in a certain time interval. The use of short segments for the calculation of the acceleration features would improve the ability to correctly recognize short activities and to measure activity duration, supposing that the classification performances are constant regardless of the segment size.

Detection of activity intensity is essential for measuring PA. According to Ainsworth et al. (1), the categorization of dynamic activities such as walking and cycling into light, moderate, or vigorous intensity depends largely on movement speed. In the past, accelerometers have been used to assess intensity of PA by measuring AAU. Several studies showed a linear relationship between AAU and activity intensity as defined by energy expenditure measured using indirect calorimetry $(13,18,22)$. However, this linearity is valid only within a single activity type (22). In addition, this AAU-based method can only be applied to activities with duration longer than $1 \mathrm{~min}$. For this reason, improvements are required to evaluate intensity of a variety of activities in daily life. Recently, trunk-mounted accelerometers have been used to assess speed of walking. Schutz et al. (27) proposed an algorithm to estimate the walking speed based on features of the acceleration signal. Other studies $(30,31)$ proposed to measure walking speed, estimating gait characteristics such as step length and duration of the stride cycle by using the acceleration of the body. Nowadays, the most accurate method to measure walking, running, and cycling speed in daily life is based on accelerometers and global positioning systems (GPS) (26). However, accurate GPS units have limited wearability and are still too expensive to be used in large-scale studies (28).

The purpose of this study was threefold. The first aim was to use one triaxial accelerometer to identify a large number of activities like walking, running, cycling, standing, sitting, and lying, developing a decision tree model. The second aim was to determine the highest time resolution achievable, preserving the classification performance of the model. For this purpose, a decision tree was developed using features measured in not overlapping intervals of different length, and the consequent classification performance was evaluated. The third aim was the definition of intensity for common dynamic activities like walking, running, and cycling, investigating the association between features of the acceleration signal and movement speed.

\section{METHODS}

Subjects. Twenty healthy adults $(13$ men and 7 women) were recruited by advertisement in local newspapers. All subjects gave written informed consent to participate in the study, which was approved by the Ethics Committee of the Maastricht University Medical Centre. Subjects' characteristics are described in Table 1.

Instrumentation. Body acceleration was measured with a modified version of "the triaxial accelerometer for movement registration" (Tracmor; Philips Research, Eindhoven, The Netherlands) as applied in previous studies for the assessment of PA $(5,23)$. Sensor and data logger were integrated in one device. The sensor detected accelerations in three perpendicular directions, and the sampling frequency of the signal was set to $20 \mathrm{~Hz}$. The battery life of the device was $36 \mathrm{~h}$ at this sampling frequency. The device size was $8 \times 3.5 \times 1 \mathrm{~cm}$, and the weight was $34.85 \mathrm{~g}$ (battery included). The device is currently used for research purposes, and it is not commercially available. During the trial, the device was attached on the lower back using an elastic belt and it was positioned under the clothes to maximize the comfort for the subject. The device was oriented to have the $x, y$, and $z$ axes, sensing acceleration in the vertical, medio-lateral, and anteroposterior direction of the body, respectively.

Experimental methods. The experimental setup consisted of series of physical activities performed in supervised conditions. The subjects were involved in the following activities: lying on a bed, sitting on a chair, sitting while working on a computer, standing, standing washing dishes, walking along a corridor, walking downstairs and walking upstairs, walking outdoors, running outdoors, and cycling (Fig. 1). The walking and running outdoors activities were conducted on a level and straight sidewalk of $226 \mathrm{~m}$. The cycling part was performed on a straight street of $428 \mathrm{~m}$. The subjects were instructed to

\begin{tabular}{lccc}
\multicolumn{4}{l}{ TABLE 1. Subjects' characteristics. } \\
\hline Parameters & All & Calibration & Validation \\
\hline $\mathrm{n}(\mathrm{M} / \mathrm{F})$ & $20(13 / 7)$ & $15(10 / 5)$ & $15(3 / 2)$ \\
Age $(\mathrm{yr})$ & $29 \pm 6$ & $29 \pm 7$ & $28 \pm 5$ \\
Weight $(\mathrm{kg})$ & $72 \pm 9.0$ & $70.5 \pm 9.9$ & $74.3 \pm 5.7$ \\
Height $(\mathrm{m})$ & $1.74 \pm 0.09$ & $1.75 \pm 0.09$ & $1.74 \pm 0.12$ \\
BMI $\left(\mathrm{kg} \cdot \mathrm{m}^{-2}\right)$ & $23.6 \pm 3.2$ & $23.1 \pm 2.7$ & $24.9 \pm 4.6$
\end{tabular}

Data are presented as mean \pm SD.

BMI, body mass index; All, characteristics of the population used for the development and for the cross-validation of the decision trees; Calibration, characteristics of the population used for the development of the models to estimate walking, running, and cycling speed; Validation, characteristics of the population used to test the performance of the models to estimate speed. 


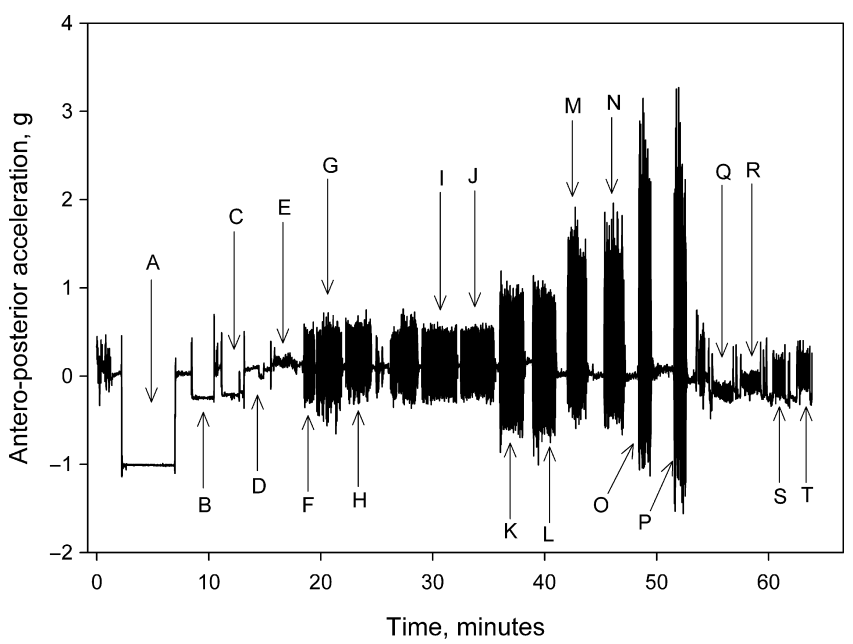

FIGURE 1-Acceleration measured in the antero-posterior direction of the body ( $z$ axis) during the experimental protocol. Arrows highlight the signal measured during the tasks included in the test: lying (A); sitting (B); sitting working on a computer (C); standing (D); washing dishes (E); walking along a corridor (F); walking downstairs (G); walking upstairs (H); walking outdoor (I-L); running outdoor (M-P); cycling outdoor $(\mathrm{Q}-\mathrm{T})$.

perform four walking, running, and cycling outdoor tasks, adopting four different speeds voluntarily chosen. Average speed was measured by dividing the path length covered by the duration of the activity task. During the cycling activities, the same bicycle with a rear wheel size of 0.71 $m$ and a gear ratio of $48 / 20$ was used by the test participants. The starting and finishing time of each activity was recorded with a stopwatch. The stopwatch and the internal clock of the accelerometer were synchronized to the same reference.

Data processing. The acceleration signal of each activity task was isolated according to the starting and the finishing time as recorded with the stopwatch. Valid data for each activity task was considered from $5 \mathrm{~s}$ after the starting time to $5 \mathrm{~s}$ before the finishing time. This 5-s time delay was set to analyze only data recorded during a stationary state for each activity, and it was determined by visual inspection of the data set. The isolated acceleration signal was then processed to calculate features to use for the development of an activity classification model. Accurate assessment of activity duration requires analysis in small segments, but segments of larger size might carry more meaningful information on the type of activity and improve the recognition of PA. To investigate which segment size allowed the highest classification accuracy, six decision trees were developed by analyzing the acceleration signal using segments of $0.4,0.8,1.6,3.2,6.4$, and $12.8 \mathrm{~s}$, including $8,16,32,64,128$, and 256 samples, respectively. The acceleration signal stored in each segment was processed to extract features in the time and frequency domain.

The acceleration features in the time domain were as follows: the average $(a)$, the standard deviation $(\sigma)$, the peak-to-peak distance $\left(a^{\mathrm{pp}}\right)$, the cross-correlation $(R)$ of the acceleration in the same axis, and the $R$ of the acceleration between sensing axes. The $R$ of the acceleration in the same axis was calculated as presented in equations 1 and 2 .

$$
\begin{aligned}
& R_{\alpha \beta}=\max \left(r_{\alpha \beta}\right) \\
& r_{\alpha \beta}(i)= \begin{cases}\sum_{j=0}^{N-i-1} \alpha_{i+j} \beta_{j,} & i \geq 0 \\
r \alpha \beta(-i), & i<0\end{cases}
\end{aligned}
$$

where $\alpha$ and $\beta$ represent two subsequent segments of the same axis, $i$ is the shift between the two segments, and $j$ is an index that covers the full length of the overlapping samples between $\alpha$ and $\beta$. $N$ represents the segment size. This feature provided a measure of the similarity in the acceleration over two subsequent time intervals for the same axis. The $R$ of the acceleration between axes was also calculated as defined in equations 1 and 2 . In this case, $\alpha$ and $\beta$ represent segments of the acceleration in the same time interval but recorded on different axes. This feature provided a measure of the similarity in the acceleration between axes. Some features were also computed in the frequency domain. Firstly, the power spectral density $(P)$ of the acceleration was used to define the harmonic content of the signal. $P$ was calculated using the fast Fourier transform algorithm on the acceleration signal of each segment. After that, attributes of $P$ were described by frequency domain features, such as the dominant frequency, the amplitude of the spectral peak $(A)$, and the frequency domain entropy $(J)$. The dominant frequency was the frequency at which $P$ had the maximum value. The $A$ was defined as the maximum value of $P$, and $J$ was defined as in equation 3 .

$$
J=-\sum_{i=1}^{N / 2}[\bar{P}(i) \log (\bar{P}(i))]
$$

where $\bar{P}$ is the normalized power spectral density, $i$ is an index that cover the entire length of $P$, and $N$ is the number of samples contained in the segment of the acceleration. These features were measured for each axis and used for the classification of the segments. The processing scripts used to calculate the features were developed using Matlab (The MathWorks, Natick, MA).

Modeling and statistics. Decision tree models were developed to identify activity types, as proposed earlier $(2,8)$. The activity tasks performed by each subject during the experimental protocol were grouped in categories addressed by the decision tree. These categories were "lie," "sit," "stand," "dynamic standing" (DS), "walk," "run," and "cycle." The lying task was labeled as "lie," the sitting and working on a computer tasks were labeled as "sit," and the standing task was labeled as "stand." The washing dishes task, dynamic standing, was labeled as "DS." The walking along a corridor, walking downstairs, walking upstairs, and walking outdoor tasks were labeled as "walk." The running and cycling tasks were labeled as "run" and "cycle," respectively. 
The development of the decision tree consisted of two main steps. The first step was the selection of the best features to use for the classification of the training data set. The second step was the definition of logical conditions based on the selected features to drive the classification of the training data set. For each segment size used to calculate acceleration features, a decision tree was developed. The classification performance of each decision tree was tested using the leave-one-subject-out cross-validation algorithm (12). The classification accuracy and the $F$-score obtained from the cross-validation were used to determine which segment size allowed the development of the decision tree with the highest classification performance. The classification accuracy was defined as the average percentage of correctly classified segments over the entire set of crossvalidation segments as measured at each step of the leaveone-subject-out cross-validation. The $F$-score was calculated as the average of the harmonic mean between sensitivity and positive predictive value measured at each step of the leave-one-subject-out cross-validation (equation 4). This parameter was defined to evaluate the overall performance of the model specifically for each activity type.

$$
F \text { - } \text { score }_{k}=\frac{1}{20} \sum_{i=1}^{20} 2 \frac{\mathrm{Se}_{k}^{i} \times \mathrm{PPV}_{k}^{i}}{\mathrm{Se}_{k}^{i}+\mathrm{PPV}_{k}^{i}}
$$

where $i$ is an index that represents each step of the leaveone-subject-out cross-validation, $k$ represents the type of activity considered, the constant 20 is the number of steps in the leave-one-subject-out cross-validation, $\mathrm{Se}$ is the sensitivity of the decision tree for the $k$-activity type, and PPV is the positive predictive value of the decision tree for the $k$-activity type. Se describes the ability to avoid falsenegative classifications for a certain activity type. PPV defines the ability to avoid false-positive classifications for a certain activity type. Considering the number of truepositive classification (TP), false-positive classification (FP), true-negative classification (TN), and false-negative classification (FN), Se was calculated as TP / (TP + FN), and PPV was calculated as TP / (TP + FP). The Student $t$-test was used to determine differences in the classification accuracy of the decision trees trained using different segment length. The classification accuracies were expressed as mean \pm SD. Statistical significance level was set to $P<0.05$. The development of the classification models and the cross-validation was performed using Matlab (The MathWorks). The C4.5 algorithm (6) was used for the training and pruning of the decision tree. The minimum number of objects included in the terminal branches of the decision tree was set to $5 \%$ of the training instances.

Three separate models were developed to estimate walking, running, and cycling speed for the outdoors tasks. Stepwise multiple linear regression was used to select the best independent elements to include in the models. The independent elements of the models were body characteristics and acceleration features measured in segments of $6.4 \mathrm{~s}$ and averaged over the entire duration of the activity task. The study population was divided in two groups: the "calibration group" (75\%) and the "validation group" $(25 \%)$. Subjects were randomly assigned to the calibration or the validation group. Data from the calibration group were used to develop the models to estimate walking, running, and cycling speed. Data from the validation group were used to test the performance of the developed models. The standard error of estimation was defined to measure the ability of the developed models in fitting the measured speed of the calibration population. The standard error of validation (SEV) was defined to determine the ability of the models in predicting the measured speed of the validation population. Furthermore, the residuals between estimated and measured speeds of the validation population were analyzed to define the bias and the limits of agreement of the models with the measurements (4). The development of these models to estimate speed and the statistical analysis of the prediction performance were conducted using Matlab statistical toolbox (The MathWorks) and SigmaStat (Systat software, San Jose, CA).

\section{RESULTS}

Activity classification. Decision trees developed using segments of 12.8 and $6.4 \mathrm{~s}$ showed the highest classification accuracy. The smaller the segments size considered the lower was the classification accuracy (Table 2). There was no significant difference between the classification accuracy of the models developed using segments of 12.8 and $6.4 \mathrm{~s}(P=0.41)$. The paired $t$-test between the classification accuracy of the models developed using segments of 3.2

TABLE 2. Classification performances of the decision trees developed using different segment sizes.

\begin{tabular}{|c|c|c|c|c|c|c|c|c|}
\hline \multirow[b]{2}{*}{ Segment Size (s) } & \multirow[b]{2}{*}{ Classification Accuracy (\%) } & \multicolumn{7}{|c|}{ F-score (\%) } \\
\hline & & Lie & Sit & Stand & DS & Walk & Run & Cycle \\
\hline 0.4 & $90.4 \pm 0.3$ & 100.0 & 85.7 & 53.9 & 67.6 & 97.3 & 99.1 & 89.3 \\
\hline 0.8 & $91.9 \pm 0.2$ & 100.0 & 86.4 & 59.6 & 71.9 & 98.3 & 99.7 & 92.2 \\
\hline 1.6 & $92.3 \pm 0.3$ & 100.0 & 86.6 & 58.0 & 72.8 & 98.8 & 99.9 & 93.3 \\
\hline 3.2 & $92.6 \pm 0.3$ & 100.0 & 86.7 & 59.7 & 72.5 & 99.1 & 100.0 & 93.4 \\
\hline 6.4 & $93.1 \pm 0.5$ & 100.0 & 87.4 & 62.4 & 75.2 & 99.2 & 100.0 & 93.9 \\
\hline 12.8 & $93.0 \pm 0.6$ & 100.0 & 86.4 & 60.0 & 74.5 & 99.5 & 100.0 & 95.1 \\
\hline
\end{tabular}

Bold numbers in the table represent the maximum value of $F$-score.

Segment size, length of the intervals used to segment the acceleration; classification accuracy, percentage of the correctly classified segments over the entire set of validation segments as measured using the leave-one-subject-out cross-validation; F-score, defined as the harmonic mean of sensitivity and positive predictive value of the classification method; DS, dynamic standing activity. 


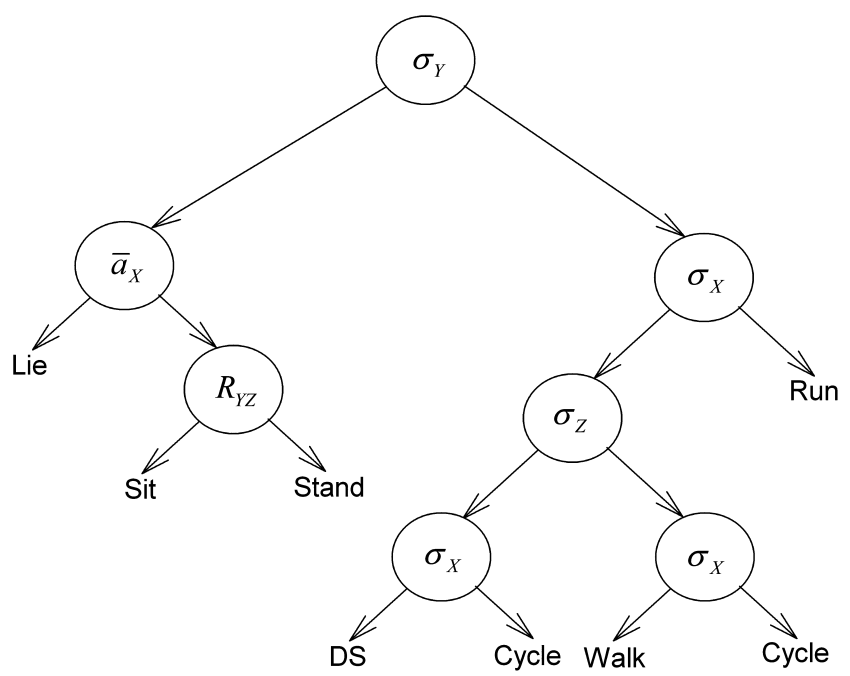

FIGURE 2-Structure of the decision tree developed using segments of $6.4 \mathrm{~s}$. The circles represent decision nodes. In the decision nodes, logic conditions based on the noted features distinguish the seven activity types (lie, sit, stand, dynamic standing [DS], walk, run, and cycle). The features selected for the classification were the standard deviation of the acceleration in the vertical, mediolateral, and anteroposterior direction $\left(\sigma_{x}, \sigma_{y}, \sigma_{z}\right)$; the average acceleration in the vertical direction of the body $\left(a_{x}\right)$; and the cross-correlation of subsequent intervals of the acceleration in the anteroposterior direction $\left(R_{z}\right)$.

and $1.6 \mathrm{~s}$ showed a $P=0.05$. The paired $t$-test between the classification accuracy of all the other models showed a statistically significant difference $(P<0.05)$. The $F$-scores of the decision tree developed using segments of $12.8 \mathrm{~s}$ for each activity types ranged from $60 \%$ to $100 \%$ (Table 2 ). The features selected to classify activity types were stable at each step of the cross-validation and were also stable for each segment length considered (Fig. 2).

Speed estimation. Subjects' characteristics for the calibration and the validation group were similar (Table 1). Measured walking, running, and cycling speeds were $5.2 \pm$
$0.9,9.8 \pm 2.2$, and $16.0 \pm 4.0 \mathrm{~km} \cdot \mathrm{h}^{-1}$, respectively. The model to estimate walking speed showed an SEE of $0.2 \mathrm{~km} \cdot \mathrm{h}^{-1}$ and an SEV of $0.47 \mathrm{~km} \cdot \mathrm{h}^{-1}$. The model had a bias of $0.32 \mathrm{~km} \cdot \mathrm{h}^{-1}$, with $\pm 95 \%$ prediction interval of $\pm 0.70 \mathrm{~km} \cdot \mathrm{h}^{-1}$ (confidence interval [CI] from -0.38 to $1.02 \mathrm{~km} \cdot \mathrm{h}^{-1}$ ). The model to estimate running speed showed an SEE of $1.26 \mathrm{~km} \cdot \mathrm{h}^{-1}$. The SEV was $2.45 \mathrm{~km} \cdot \mathrm{h}^{-1}$. This model overestimated the measured speed with a bias of $0.29 \mathrm{~km} \cdot \mathrm{h}^{-1}$, with $\pm 95 \%$ prediction interval of \pm 4.89 $\mathrm{km} \cdot \mathrm{h}^{-1}$ (CI from -4.6 to $5.18 \mathrm{~km} \cdot \mathrm{h}^{-1}$ ). The model to estimate cycling speed showed an SEE of $1.36 \mathrm{~km} \cdot \mathrm{h}^{-1}$. The SEV was $2.88 \mathrm{~km} \cdot \mathrm{h}^{-1}$. This model had a bias of $0.26 \mathrm{~km} \cdot \mathrm{h}^{-1}$, with $\pm 95 \%$ prediction interval of $\pm 5.76 \mathrm{~km} \cdot \mathrm{h}^{-1}$ (CI from -5.50 to $6.02 \mathrm{~km} \cdot \mathrm{h}^{-1}$ ). The independent elements of these three models are presented in Table 3.

\section{DISCUSSION}

Classification of PA. In this study, we developed a method to identify types of PA. The classification process was based on information carried by features of the acceleration of the body as measured using one triaxial accelerometer placed on the lower back. On the basis of this information, a decision tree showed high accuracy in identifying activity types. The highest classification accuracy $(93 \%)$ was achieved by measuring acceleration features in intervals of 6.4 or $12.8 \mathrm{~s}$.

During the experimental protocol, 20 standardized activities were performed by the subjects. These activities were grouped in seven classes that represent common types of daily PA. The lying, sitting, and standing classes were defined to represent human postures. The walking and running classes were defined to represent gait and ambulation. The DS class was defined to represent human movements performed in the standing position not related

TABLE 3. Models to estimate walking, running, and cycling speed.

\begin{tabular}{|c|c|c|c|c|}
\hline Var & Coef & SE & Partial $-r^{2}$ & $P$ \\
\hline \multicolumn{5}{|c|}{ Walking speed $\left(r^{2}=96.3 \%\right)$} \\
\hline Int & 55.18 & 13.06 & & \\
\hline $\ln \left(\sigma_{x}\right)$ & 2.29 & 0.08 & 84.5 & $<0.001$ \\
\hline$R_{y z}$ & $2.65 \times 10^{-8}$ & $0.24 \times 10^{-8}$ & 89.5 & $<0.001$ \\
\hline$H^{H 2}$ & 2.42 & 0.30 & 93.8 & $<0.001$ \\
\hline $\bar{a}_{x}$ & -0.03 & 0.0057 & 95.2 & $<0.001$ \\
\hline$J_{x}$ & -0.13 & 0.03 & 96.3 & $<0.001$ \\
\hline \multicolumn{5}{|c|}{ Running speed $\left(r^{2}=70.2 \%\right)$} \\
\hline Int & -53.76 & & & \\
\hline$a_{z}^{\mathrm{pp}}$ & 0.006 & $9.39 \times 10^{-4}$ & 53.5 & $<0.001$ \\
\hline$R_{z x}$ & $8.88 \times 10^{-8}$ & $1.68 \times 10^{-8}$ & 64.1 & $<0.001$ \\
\hline$A_{z}$ & $-7.81 \times 10^{-6}$ & $3.33 \times 10^{-6}$ & 67.3 & 0.023 \\
\hline$A_{y}$ & $5.36 \times 10^{-6}$ & $1.97 \times 10^{-6}$ & 70.2 & 0.009 \\
\hline \multicolumn{5}{|c|}{ Cycling speed $\left(r^{2}=86.6 \%\right)$} \\
\hline Int & 4.76 & & & \\
\hline$\sigma_{z}$ & 0.33 & 0.03 & 78.7 & $<0.001$ \\
\hline$f_{z}$ & 1.01 & 0.26 & 85.0 & $<0.001$ \\
\hline$\sigma_{x}$ & 0.06 & 0.02 & 86.6 & 0.012 \\
\hline
\end{tabular}

$r^{2}$, correlation coefficient; SEE, standard error of estimates; Var, acceleration features selected as predictor variables in the models; Coef, regression coefficients of the variables included in the models; SE, standard error of the regression coefficients; Partial $-r^{2}$, partial $r^{2}$ of the regression model; $P$, observed level of statistical significance; Int, intercept of the regression line; $\operatorname{In}\left(\sigma_{x}\right)$, natural logarithm of the SD ( $x$ axis); $R_{y z}$, cross-correlation between the acceleration measured in the mediolateral and anteroposterior directions $(y$ and $z$ axes); $H$, body height; $\bar{a}_{x}$, average value of the acceleration ( $x$ axis); $J_{x}$, frequency-domain entropy ( $x$ axis); $a_{z}^{\text {pp }}$, peak-to-peak distance ( $z$ axis); $R_{z x}$, cross-correlation between the acceleration measured in the anteroposterior and vertical directions ( $z$ and $x$ axes); $A_{z}$ and $A_{y}$, amplitude of the power spectral density peak ( $z$ and $y$ axes); $\sigma_{z}$ and $\sigma_{x}$, standard deviation ( $z$ and $x$ axes); $J_{z}$, dominant frequency of the power spectral density ( $z$ axis). 
TABLE 4. Classification performance of different models to identify types of PA.

\begin{tabular}{|c|c|c|c|c|c|c|c|}
\hline & Sensors & Lie (\%) & Sit (\%) & Stand $(\%)$ & Walk (\%) & Run (\%) & Cycle (\%) \\
\hline Zhang et al. (29) & 5 & 99 & 99 & 99 & 99 & 99 & NC \\
\hline Bao and Intille (2) & 5 & 95 & 95 & 96 & 90 & 88 & 96 \\
\hline Foerster et al. (10) & 4 & 89 & 100 & 88 & 99 & NC & 100 \\
\hline Ermes et al. (8) & 2 & 99 & $\mathrm{NC}^{a}$ & $\mathrm{NC}^{a}$ & 81 & 90 & 91 \\
\hline Karantonis et al. (15) & 1 & 74 & NC & $\mathrm{NC}$ & 90 & NC & NC \\
\hline Mathie et al. (21) & 1 & 99 & $\mathrm{NC}^{a}$ & $\mathrm{NC}^{a}$ & 100 & NC & NC \\
\hline Current study & 1 & 100 & 85 & 59 & 99 & 100 & 96 \\
\hline
\end{tabular}

Sensors, number of sensors used to measure body acceleration; \% values, represent sensitivity of the model developed to identify activity types; current study, sensitivity data relative to the decision tree developed using segments of $6.4 \mathrm{~s}$; NC, not considered.

${ }^{a}$ Sitting and standing were defined as a single activity class, and the classification accuracy was of $95 \%$.

to ambulation. In addition, the cycling class was considered because cycling is a popular means of transport for trips of short distance in several countries (25).

A close examination of the structure of the decision tree developed using segments of $6.4 \mathrm{~s}$ permitted to understand the role of the different features in the classification process (Fig. 2). The standard deviation of the acceleration in the mediolateral direction $\left(\sigma_{y}\right)$ was the feature used to group in one branch activities as "lie," "sit," and "stand" and to group in the other branch activities as "DS," "walk," "run," and "cycle." Thus, the standard deviation of the acceleration in this direction of the body acquired high value for dynamic or ambulatory movements while it attained low values for static activities. The orientation of the vertical direction of the body with respect to the direction of gravity $\left(a_{x}\right)$ was the feature used to identify lying. The cross-correlation between subsequent time intervals of the antero-posterior acceleration $\left(R_{z}\right)$ was used to identify sitting and standing. A high value of the SD $\left(\sigma_{x}\right)$ of the acceleration in the vertical direction of the body was characteristic of running. Classification of the "walk," "cycle," and "DS" types was mainly determined by the $\operatorname{SD}\left(\sigma_{z}, \sigma_{x}\right)$ in the antero-posterior and the vertical direction of the body. The decision tree showed high performance for identifying lying, sitting, walking, running, and cycling because the $F$-score associated to these activities was above $86 \%$ in the models based on segments of 6.4 and $12.8 \mathrm{~s}$. Thus, appropriate acceleration features were considered to classify these activities. Standing and DS were frequently misclassified by the decision tree, and this was reflected by the relatively low $F$-score. The reason was that washing dishes was a standing activity characterized by acceleration of small magnitude, and therefore there were no features able to clearly discriminate the acceleration of these two activities.

The identification of lying, walking, running, and cycling, using the decision tree developed with segments of $6.4 \mathrm{~s}$, showed an accuracy comparable to that obtained in other studies where PA was measured using several sensors positioned in different body parts (Table 4). However, the use of one accelerometer presented a limited ability to identify the sitting and standing postures. The decision trees trained using segments of 6.4 and $12.8 \mathrm{~s}$ showed an elevated classification accuracy as compared with the one obtained in earlier studies $(8,15,24)$. However, it is worth to notice that in the current study, the acceleration signal of each activity type had different duration. According to the test protocol, a greater number of walking, running, and cycling segments were collected as compared with the number of sitting, standing, and DS segments. In view of the fact that walking, running, and cycling were identified more easily by the decision tree than sitting, standing, and DS, the disproportion in the number of segments per activity type determined a general increase of the classification accuracy. Furthermore, the fact that the DS class was represented by one activity task in the experimental protocol could be recognized as a limitation of this study because there is a broad range of household and lifestyle-related activities that might be considered to define this category.

Assessment of activity duration. The accurate assessment of activity duration is important for the correct evaluation of individuals' behavior. In free-living conditions, many activities are discontinuous and have a short duration; for instance, the daily walking activity comprises many short bouts as pointed out in a recent investigation by Levine et al. (19). The assessment of activity duration requires the correct detection of the instants at which an activity starts and finishes. The approach adopted by decision trees for the identification of activity type, which is based on segment by segment analysis, encumbers the exact detection of the activity boundaries. In natural conditions, the segmentation would rarely match with the beginning or the end of an activity. Therefore, the performances of the classification model at the boundaries of an activity are unpredictable because the segment will present partly the features of the previous or of the following activity and partly the features of the activity under investigation. This boundary ambiguity of the acceleration features might generate a misclassification, and consequently the activity duration can be incorrectly estimated. In addition, activities of duration smaller than the segment size may be misclassified as the information contained in the measured features will belong not only to the considered activity but also to the adjacent parts. However, these errors are minimized if the segment has a short length. Thus, the advantages offered by the use of short intervals for the segmentation of the acceleration signal are the reduced error in the definition of activity duration and the increased accuracy in the classification of short activities. When the segmentation of the acceleration signal is made by considering contiguous intervals, the use 
of short intervals would increase the time resolution of analysis, which improves the detection of activity duration. Another method used to increase the time resolution is the segmentation of the signal in overlapping intervals for the calculation of acceleration features. Using this technique, the time resolution is determined by the level of overlap between segments, and it can be increased without reducing the segment size. However, because of the overlap, misclassifications due to activity transitions would affect more segments. Thus, the shorter the segments of analysis, the lower the propagation of the classification error due to activity transitions, improving in this way the definition of activity duration.

In this study, we reported that the use of short intervals for the computation of acceleration features led to a reduction of the classification accuracy. Using features measured in segments of 0.4 s reduced by $3 \%$ the classification accuracy as compared with the one obtained with segments of 6.4 and $12.8 \mathrm{~s}$. The decline of classification performance for small segments of analysis concerned most of the activity types as shown by the decrease of the $F$-score. This can be explained by the fact that the features had a higher intraclass variability (variability within the same activity class) when computed in shorter segments. Given that decision trees discriminate features belonging to different activities by defining cutoff values, if the features have higher intraclass variability, the risk of overlapping between values of different activities is higher. This reduces the ability of the cutoff values to distinguish activity types, which results in a decrease in the classification accuracy of the decision tree. However, the accuracy to identify PA was similar for the model with time resolution of $6.4 \mathrm{~s}$ as compared with the model with time resolution of $12.8 \mathrm{~s}$. Thus, segmenting the acceleration signal in nonoverlapping windows of $6.4 \mathrm{~s}$ gave the highest time resolution for measuring activity duration without decreasing the classification accuracy.

Estimation of activity intensity. Intensity of walking, running, and cycling is largely determined by speed. According to Ainsworth et al. (1), movement speed could be used to categorize walking, running, and cycling in intensity levels, such as light $(\mathrm{MET}<3)$, moderate $(3<$ MET $<6$ ), or vigorous (MET $>6$ ). For example, walking below $4 \mathrm{~km} \cdot \mathrm{h}^{-1}$ could be defined as light-intensity activity.

\section{REFERENCES}

1. Ainsworth BE, Haskell WL, Whitt MC, et al. Compendium of physical activities: an update of activity codes and MET intensities. Med Sci Sports Exerc. 2000;32(9 suppl):S498-516.

2. Bao L, Intille SS. Activity recognition from user-annotated acceleration data. Pervasive Comput. 2004;3001:1-17.

3. Blair SN, Cheng Y, Holder JS. Is physical activity or physical fitness more important in defining health benefits? Med Sci Sports Exerc. 2001;33(6 suppl):S379-99.

4. Bland JM, Altman DG. Statistical methods for assessing agreement between two methods of clinical measurement. Lancet. 1986;1(8476):307-10.
Walking between 4 and $7.2 \mathrm{~km} \cdot \mathrm{h}^{-1}$ can be defined as moderate-intensity activity, and walking above $7.2 \mathrm{~km} \cdot \mathrm{h}^{-1}$ could be defined as vigorous-intensity activity (1). Furthermore, a linear association was observed between walking, running, and cycling speed and energy expenditure. The slope of the linear regression describing these relationships was estimated to be $0.8,0.77$, and $0.66 \mathrm{MET} \cdot \mathrm{h} \cdot \mathrm{km}^{-1}$ for walking between 3 and $5 \mathrm{~km} \cdot \mathrm{h}^{-1}$, for running, and for cycling, respectively, $(1,9)$. In this study, acceleration features and body characteristics were used to develop models to estimate walking, running, and cycling speed. The use of these models in intervals of the acceleration identified as walking, running, or cycling allows the evaluation of activity intensity specific for the type of PA. This approach seems to be in line with the method proposed by Crouter et al. (7) to improve the prediction accuracy of energy expenditure using accelerometer output. Compared with GPS estimates, the proposed models had lower accuracy. However, the SEE of the walking speed model was still similar to the one achieved in models based on GPS measurements (26).

The applicability in a natural condition of the cycling speed estimation might present limitations. The reason is that during the experimental protocol, only one bicycle and one specific gear was used by the subjects. Therefore, the performance of the model should be tested on different bicycles to confirm the validity of the estimation properties. Furthermore, the limitation of this method for the assessment of activity intensity was that the effect of walking, running, or cycling on slopes was not considered. Hence, the intensity of moving uphill or downhill might be incorrectly evaluated.

\section{CONCLUSIONS}

This study demonstrated the ability of a triaxial accelerometer in detecting type, duration, and intensity of PA by using models based on acceleration features. Future studies are needed to validate the presented models in free-living conditions and in specific populations like children, elderly, and obese subjects.

This study was funded by Philips Research. The results of the present study do not constitute endorsement by the American College of Sports Medicine. 
uncontrolled conditions. IEEE Trans Inf Technol Biomed. 2008; 12:20-6.

9. Falls HB, Humphrey LD. Energy cost of running and walking in young women. Med Sci Sports Exerc. 1976;8(1):9-13.

10. Foerster F, Smeja M, Fahrenberg J. Detection of posture and motion by accelerometry: a validation study in ambulatory monitoring. Comput Hum Behav. 1999;15(5):571-83.

11. Haskell WL, Lee IM, Pate RR, et al. Physical activity and public health: updated recommendation for adults from the American College of Sports Medicine and the American Heart Association. Med Sci Sports Exerc. 2007;39(8):1423-34.

12. Hastie T, Tibshirani R, Friedman J. The Elements of Statistical Learning. Springer; 2001. 214 p.

13. Hendelman D, Miller K, Bagget C, Debold E, Freedson P. Validity of accelerometry for the assessment of moderate intensity physical activity in the field. Med Sci Sports Exerc. 2000; 32(9 suppl):S442-9.

14. Jebb SA, Moore MS. Contribution of a sedentary lifestyle and inactivity to the etiology of overweight and obesity: current evidence and research issues. Med Sci Sports Exerc. 1999;31(11 suppl): S534-41.

15. Karantonis DM, Narayanan MR, Mathie M, Lovell NH, Celler BG. Implementation of a real-time human movement classifier using a triaxial accelerometer for ambulatory monitoring. IEEE Trans Inf Technol Biomed. 2006;10(1):156-67.

16. Kern N, Schiele B, Schmidt A. Multi-sensor activity context detection for wearable computing. In: European Symposium on Ambient Intelligence; 2003 Nov 3-4: The Netherlands; 2003. p. 220-32.

17. Kriska AM, Saremi A, Hanson RL, et al. Physical activity, obesity, and the incidence of type 2 diabetes in a high-risk population. Am J Epidemiol. 2003;158(7):669-75.

18. Levine JA, Baukol PA, Westerterp KR. Validation of the Tracmor triaxial accelerometer system for walking. Med Sci Sports Exerc. 2001;33(9):1593-7.

19. Levine JA, McCrady SK, Lanningham-Foster LM, Kane PH, Foster RC, Manohar CU. The role of free-living daily walk- ing in human weight gain and obesity. Diabetes. 2008;57(3): 548-54.

20. Macfarlane DJ, Lee CCY, Ho EYK, Chan KL, Chan D. Convergent validity of six methods to assess physical activity in daily life. J Appl Physiol. 2006;101(5):1328-34.

21. Mathie MJ, Celler BG, Lovell NH, Coster ACF. Classification of basic daily movements using a triaxial accelerometer. Med Biol Eng Comput. 2004;42(5):679-87.

22. Midorikawa T, Tanaka S, Kaneko K, et al. Evaluation of lowintensity physical activity by triaxial accelerometry. Obesity. 2007;15:3031-8.

23. Plasqui G, Westerterp KR. Physical activity assessment with accelerometers: an evaluation against doubly labeled water. Obesity. 2007;15:2371-9.

24. Pober DM, Staudenmayer J, Raphael C, Freedson PS. Development of novel techniques to classify physical activity mode using accelerometers. Med Sci Sports Exerc. 2006;38(9):1626-34.

25. Rietveld P, Daniel V. Determinants of bicycle use: do municipal policies matter? Transp Res Part A Policy Pract. 2004;38(7): 531-50.

26. Schutz Y, Herren R. Assessment of speed of human locomotion using a differential satellite global positioning system. Med Sci Sports Exerc. 2000;32(3):642-6.

27. Schutz Y, Weinsier S, Terrier P, Durrer D. A new accelerometric method to assess the daily walking practice. Int J Obes. 2002; 26(1):111-8.

28. Tan HL, Wilson AM, Lowe J. Measurement of stride parameters using a wearable GPS and inertial measurement unit. $J$ Biomech. 2008;41(7):1398-406.

29. Zhang K, Werner P, Sun M, Pi-Sunyer FX, Boozer CN Measurement of human daily physical activity. Obes Res. 2003; 11(1):33-40.

30. Zijlstra W. Assessment of spatio-temporal parameters during unconstrained walking. Eur J Appl Physiol. 2004;92(1-2):39-44.

31. Zijlstra W, Hof AL. Assessment of spatio-temporal gait parameters from trunk accelerations during human walking. Gait Posture. 2003;18(2):1-10. 